\title{
PREMEDICACION ANESTESICA CON MIDAZOLAM EN NIÑOS EN EL HOSPITAL REGIONAL DE ICA, PERÚ. 2007-2008
}

\author{
María Luz Mateo-Corrales ${ }^{1,2, a}$ \\ ${ }^{1}$ Facultad de Medicina. Universidad Nacional San Luis Gonzaga. Ica. Perú. \\ ${ }^{2}$ Hospital San Juan de Dios. Pisco, Ica, Perú. \\ ${ }^{a}$ Médico Anestesiólogo.
}

\section{RESUMEN}

Objetivo: Evaluar las ventajas de la premedicación anestésica con Midazolam oral Vs Midazolam intramuscular (IM) en niños de 1 -7 años, en el Hospital Regional de Ica. Material y Métodos: Se realizó un estudio experimental, tipo Ensayo Clínico controlado, ciego simple, con asignación alternada. Se incluyeron 120 niños, entre 1 y 7 años, divididos en 2 grupos comparables en edad, riesgo anestésico y cirugía propuesta. Grupo A $(n=60)$ estudio, con Midazolam oral y grupo $B(n=60)$ control, con Midazolam IM. Resultados: La edad media fue de $5.57 \pm 1.42$ años para el grupo midazolam oral y $4.95 \pm 1.36$ años para el grupo midazolam IM. Al evaluar el llanto luego de la premedicación: en el grupo midazolam oral: $3,33 \%$ llanto moderado y $96,67 \%$ sin llanto y en el grupo midazolam IM: $100 \%$ no presentaron Ilanto. (p> 0.05). Somnolencia: luego de la premedicación con Midazolam oral se encontró 6,67\% despiertos, quietos y 93,33 somnolientos mientras que en el grupo Midazolam IM $15 \%$ despiertos, quietos y $85 \%$ somnolientos. ( $p>0.05$ ). Movimiento: luego de premedicación con midazolam oral $3,14 \%$ con movimientos fuertes que dificultaron el tratamiento, $85 \%$ con ligero movimiento que no interfirieron con el tratamiento, $11,7 \%$ sin movimiento y midazolam IM 98,30\% con movimiento ligero no interfería con el tratamiento, $1,70 \%$ no presentó movimiento. $(p<0,05)$. Conclusiónes: El Midazolam vía oral es una buena alternativa para la premedicación en pediatría. La cualidad resaltante es no aumentar el estrés por ser su administración indolora.

Palabras clave: Medicación Preanestésica, Midazolam, sedación consciente. (fuente: DeCS BIREME)

\section{PREMEDICATION WITH MIDAZOLAM ANESTHESIA IN CHILDREN IN THE REGIONAL HOSPITAL IN ICA, PERU 2007-2008}

\begin{abstract}
Objective: To Evaluate the advantages of anesthetic premedication with oral midazolam vs midazolam intramuscular (IM) in children 1 -7 years, in the Regional Hospital .Material and Methods: It was realized an experimental study, controlled clinical trial type, single-blind, with alternate allocation. We included 120 children between 1 and 7 years, divided in 2 groups were comparable in age, anesthetic risk and surgery proposal. Group $A(n=60)$ study, with oral midazolam and group B ( $n=60)$ control, with IM midazolam. Results: The mean age was $5.57 \pm 1.42$ years for the group with oral midazolam and $4.95 \pm 1.36$ years for the group IM midazolam. In assessing the crying after premedication: in the group oral midazolam: $3.33 \%$ crying and moderate $96.67 \%$ without crying and in the midazolam group IM: $100 \%$ were not crying. (P> 0.05 ) Drowsiness: after premedication with oral midazolam was found $6.67 \%$ awake, motionless and 93.33 drowsy while in the midazolam group IM $15 \%$ awake, motionless and $85 \%$ drowsy. ( $P>0.05$ ) Movement: after premedication with oral midazolam $3.14 \%$ with strong movements that hampered the treatment, $85 \%$ with slight movement that did not interfere with the treatment, $11.7 \%$ without movement and midazolam IM $98.30 \%$ with slight movement did not interfere with the treatment, $1.70 \%$ not presented movement. $(P<0.05)$ Conclusion: The Midazolam orally is a good alternative for premedication in pediatrics. The quality is equally important not to increase the stress by being his administration painless.
\end{abstract}

Key words: Premedication, Midazolam, Conscious sedation. (source: MeSH NLM)

\section{INTRODUCCIÓN}

Es sabido que el ingreso hospitalario para un tratamiento quirúrgico puede ocasionar trastornos emocionales en los niños, que en la mayoría de los casos no están preparados para enfrentar esa experiencia, y que puede llegar a ser traumática $^{(1,2)}$.

La visita y pre medicación anestésica debe reducir la ansiedad, bloquear reflejos autonómicos para lograr una inducción de la anestesia atraumática. En el área pediatrica, la preparación farmacológica está destinada a disminuir la respuesta al stress a los procedimientos anestésico-quirúrgicos. La vía de administración ideal para la premedicación anestésica en Pediatría es aquella que no aumente el trauma a la situación de angustia, en la que por lo general se encuentran los niños.

Castro Jerí (3) 2002 en Perú. Compara la efectividad del Midazolam en niños: vía oral y vía intranasal; encontrando que los signos vitales se mantuvieron estables, no se presentaron reacciones adversas en ninguno de los casos. Este estudio demostró que ambas vías eran de eficacia 
similar en el tratamiento dental en niños en edad preescolar.

Amundaraín et al ${ }^{(4)}, 2004$, estudió la efectividad del jarabe de Midazolam para premedicación oral en pediatría, concluyendo que el midazolam es beneficioso; el comportamiento de los niños expuestos y sus representantes fue de excelente cooperación, contándose con pacientes que se desprenden con mayor facilidad de su entorno, aceptan las maniobras de la inducción anestésica en cirugías posteriores, minimizando así el miedo y la ansiedad.

Suarez ${ }^{(5)}$, en el 2010, estudió la premedicación con Midazolam en gomitas en niños, determinando ventajas de la premedicación en éstos pacientes. Afirmando que su principal ventaja es la aceptación satisfactoria en todos los niños; teniendo una actitud deseada en el momento de la separación de sus padres; no existiendo cambios hemodinámicos significativos; aunque el despertar no fue el deseado en la mayoría de los niños.

En el área de anestesia pediátrica de nuestro hospital se utilizaba para la premedicación anestésica el Midazolam IM que muchas veces aumentaba el trauma debido al miedo que los niños tenían a las agujas, ocasionando que su hospitalización fuera una experiencia desagradable; presentando un rechazo posterior al hospital.

La realización del presente trabajo sirvió para comparar la premedicación anestésica con Midazolam, vía oral vs intramuscular y determinar si existiera o no diferencias ventajosas, en niños de 1-7 años, como alternativa eficaz para sedación consiente atraumática pre-operatoria, en procedimientos quirúrgicos de la Unidad de Cirugía Pediátrica, en el Centro Quirúrgico del Hospital Regional de Ica.

\section{MATERIAL Y MÉTODOS}

Se realizó un estudio Experimental, tipo Ensayo clínico controlado, ciego simple. Respetando los criterios de inclusión y exclusión con asignación alternada, hasta completar un total de 60 niños por grupo. Seleccionado el caso se obtuvo el consentimiento informado que fue autorizado y firmado por los padres. Se formaron 2 grupos: el grupo $A$, de estudio, recibió premedicación vía oral y el grupo $\mathrm{B}$ o control recibió pre medicación vía intramuscular.

Para el grupo A se utilizó la ampolla de Midazolam de 15 mg con volumen de $3 \mathrm{~cm}^{3}$, por su sabor amargo se le agregó $4 \mathrm{~cm}^{3}$ de jugo de manzana como edulcorante, por $\mathrm{cm}^{3}$ midazolam. El midazolam oral se administró a dosis de $0,5 \mathrm{mg} / \mathrm{kg}$.Para el grupo B se utilizó midazolam IM a dosis de $0,2 \mathrm{mg} / \mathrm{kg}$.

Luego de la aceptación se procedió a tomar las funciones vitales antes de la premedicación, posteriormente se administró el midazolam 30 minutos antes de la intervención quirúrgica. La evaluación de los niños fue antes, durante y después de la premedicación, observando los efectos en el grupo de estudio y control.

Los datos fueron obtenidos mediante la observación directa y evaluación monitorizada, no invasiva, con monitor cinco parámetros de variables hemodinámicas.
En todos los niños se evaluó: el nivel de sedación mediante la Escala de Ramsay, con 6 categorías: ansioso, colaborador, responde a mínimos estímulos, dormido pero responde rápidamente a estímulos luminosos o auditivos, dormido pero responde perezosamente a estímulos auditivos y no responde a estímulos; conducta a través de la Escala de Houpt que evalúo el llanto, movimiento y somnolencia. Así como tambien la respuesta psicológica y variables hemodinámicas como frecuencia cardiaca, frecuencia respiratoria, presión arterial y saturación de oxígeno durante la pre-inducción, anestesia y recuperación.

Por otro lado se anotaron los efectos adversos. Una vez obtenida la información se confeccionó una base de datos con el Programa SPSS versión 12. Para el análisis bivariado se utilizó la Prueba exacta de Fisher y Chi cuadrado para evaluar variables cualitativas y para variables numéricas se consideró la Prueba T de Student.

\section{RESULTADOS}

En total fueron 60 niños por grupo, 35 hombres y 25 mujeres para cada grupo, comprendidos entre 1-7 años, con edad media de $5,57 \pm 1,42$ años para el grupo $A$ y de $4,95 \pm 1,36$ años para el grupo $B$.

Luego de la premedicación evaluamos el llanto: En el grupo de Midazolam vía oral: 2 niños (3,33\%) presentaron llanto moderado y 58 niños $(96,67 \%)$ no presentaron llanto. En el grupo de Midazolam intramuscular: los 60 niños (100\%) no presentaron llanto $(p>0,05)$. (Tabla 1$)$.

Tabla 1. Comparación del llanto después de la premedicación en los niños de 1-7 años, grupo Midazolam oral (VO) vs Midazolam intramuscular (IM).

\begin{tabular}{ccccccc}
\hline $\begin{array}{c}\text { Llanto } \\
\text { después de } \\
\text { pre }\end{array}$ & \multicolumn{2}{c}{$\begin{array}{c}\text { Midazolam } \\
\text { VO }\end{array}$} & \multicolumn{2}{c}{$\begin{array}{c}\text { Midazolam } \\
\text { IM }\end{array}$} & \multicolumn{2}{c}{ Total } \\
\cline { 2 - 7 } $\begin{array}{c}\text { medicación } \\
\text { Intermitente }\end{array}$ & $\mathbf{N}$ & $\%$ & $\mathbf{N}$ & $\%$ & $\mathbf{N}$ & $\%$ \\
Moderado & 2 & 3,33 & 0 & 0,00 & 2 & 1,67 \\
No hay llanto & 58 & 96,67 & 60 & 100,00 & 118 & 98,33 \\
$\quad$ Total & 60 & 100,00 & 60 & 100,00 & 120 & 100,00 \\
\hline Fisher 0,188 & & & & & &
\end{tabular}

Fisher 0,188

Luego de la premedicación, evaluamos la somnolencia: Los niños que estuvieron inquietos disminuyeron de 26 a 4 en el grupo midazolam oral y de 39 a 9 en el grupo Midazolam intramuscular (IM) (Tabla 2).

Al evaluar la respuesta psicológica a la premedicacion, u obtuvo en el grupo midazolam oral 38 niños (63,33\%) que presentaron miedo al daño corporal y en el grupo control 60 niños $(100 \%)$ presentaron este resultado. $(p<0,05)$ (Tabla 3$)$

En cuanto al inicio del efecto de la premedicación se encontró: en el grupo A 26 niños (43,33\%) presentaron un tiempo de latencia de 15 minutos y el resto de 20 minutos. En el grupo B: 46 niños $(76,67 \%)$ lo inició a los 15 minutos y 14 niños $(23,33 \%)$ a los 20 minutos $(p<0,05)$. El inicio de efectos por vía oral fue más lento que por vía intramuscular (Tabla 4). 
Tabla 2. Comparación de la somnolencia después de la premedicación en los niños de 1-7 años, grupo Midazolam oral (VO) vs Midazolam intramuscular (IM).

\begin{tabular}{|c|c|c|c|c|c|c|}
\hline \multirow{2}{*}{$\begin{array}{c}\text { Somnolencia } \\
\text { después de } \\
\text { pre } \\
\text { medicación }\end{array}$} & \multicolumn{3}{|c|}{$\begin{array}{c}\text { Midazolam } \\
\text { VO } \\
\end{array}$} & \multicolumn{2}{|c|}{$\begin{array}{c}\text { Midazolam } \\
\text { IM } \\
\end{array}$} & Total \\
\hline & $\mathbf{N}$ & $\%$ & & $\%$ & $\mathbf{N}$ & $\%$ \\
\hline $\begin{array}{l}\text { Intermitente } \\
\text { Moderado }\end{array}$ & 4 & 6,67 & 9 & 15,00 & 13 & 10,83 \\
\hline No hay llanto & 56 & 93,33 & 51 & 85,00 & 107 & 89,17 \\
\hline Total & 60 & 100,00 & 60 & 100,00 & 120 & 100,00 \\
\hline
\end{tabular}

Tabla 3. Comparación de la respuesta psicológica a la premedicación en los niños de 1-7 años, grupo Midazolam oral (VO) vs Midazolam intramuscular (IM).

\begin{tabular}{cccccccc}
\hline $\begin{array}{c}\text { Respuesta } \\
\text { psicológica a } \\
\text { la pre }\end{array}$ & $\begin{array}{c}\text { Midazolam } \\
\text { VO }\end{array}$ & \multicolumn{2}{c}{$\begin{array}{c}\text { Midazolam } \\
\mathbf{I M}\end{array}$} & \multicolumn{2}{c}{ Total } \\
\cline { 2 - 7 } $\begin{array}{c}\text { medicación } \\
\text { Intermitente }\end{array}$ & $\mathbf{N}$ & $\%$ & $\mathbf{N}$ & $\%$ & $\mathbf{N}$ & $\%$ \\
Moderado & 98 & 63,33 & 60 & 100,00 & 98 & 81,66 \\
No hay llanto & 22 & 36,67 & 0 & 0,00 & 22 & 18,34 \\
$\quad$ Total & 60 & 100,00 & 60 & 100,00 & 120 & 100,00 \\
\hline
\end{tabular}

Tabla 4. Comparación Inicio de efecto en Tiempo de la premedicación en los niños de 1-7 años, grupo Midazolam oral (VO) vs Midazolam intramuscular (IM).

\begin{tabular}{ccccccc}
\hline $\begin{array}{c}\text { Inicio } \\
\text { efecto }\end{array}$ & $\begin{array}{c}\text { Midazolam } \\
\text { Vo }\end{array}$ & \multicolumn{2}{c}{$\begin{array}{c}\text { Midazolam } \\
\text { IM }\end{array}$} & \multicolumn{2}{c}{ Total } \\
\cline { 2 - 7 } & $\mathbf{N}$ & $\%$ & $\mathbf{N}$ & $\%$ & $\mathbf{N}$ & $\%$ \\
$\begin{array}{c}\text { Intermitente } \\
\text { Moderado }\end{array}$ & 26 & 43,33 & 46 & 76,67 & 72 & 60,00 \\
$\begin{array}{c}\text { No hay } \\
\text { Ilanto }\end{array}$ & 34 & 56,67 & 14 & 23,33 & 48 & 40,00 \\
$\quad$ Total & 60 & 100,00 & 60 & 100,00 & 120 & 100,00 \\
\hline$X^{2}=13,88$ (Chi cuadrado) & $p=0,00019$ (p value) & &
\end{tabular}

Al evaluar la sedación consiente luego de la premedicación, se obtuvo más niños tranquilos y colaboradores en el grupo Midazolam intramuscular. ( $p>0,05)$. (Tabla 5).

En ninguna medición se obtuvieron diferencias significativas en la frecuencia cardiaca (Tabla $N^{\circ} 6$ )

\section{DISCUSIÓN}

El Midazolam es una benzodiacepina utilizada en premedicación anestésica de pacientes pediátricos y adultos en muchos centros quirúrgicos hospitalarios incluyendo el Hospital Regional de Ica.

En nuestro trabajo los valores alcanzados en los parámetros llanto, somnolencia antes y después de la premedicacion no difieren significativamente en ambas vías
Tabla 5. Comparación de sedación consciente Postpremedicación en los niños de 1-7 años, grupo Midazolam oral (VO) vs Midazolam intramuscular (IM).

\begin{tabular}{ccccccc}
\hline $\begin{array}{c}\text { Inicio } \\
\text { efecto }\end{array}$ & \multicolumn{2}{c}{$\begin{array}{c}\text { Midazolam } \\
\text { VO }\end{array}$} & $\mathbf{N}$ & $\%$ & $\begin{array}{c}\text { Midazolam } \\
\text { IM }\end{array}$ & \multicolumn{2}{c}{ Total } \\
\cline { 2 - 6 } $\begin{array}{c}\text { Ansioso } \\
\text { agitado }\end{array}$ & 2 & 3,33 & 0 & 0 & 2 & 1,67 \\
$\begin{array}{c}\text { Tranquilo } \\
\text { colaborador }\end{array}$ & 54 & 90,00 & 56 & 93,33 & 110 & 91,66 \\
$\begin{array}{c}\text { Abre los ojos } \\
\text { a la orden }\end{array}$ & 4 & 56,67 & 4 & 6,67 & 8 & 6,67 \\
$\quad$ Total & 60 & 100,00 & 60 & 100,00 & 120 & 100,00 \\
\hline$p=0,36$ & & & & & &
\end{tabular}

Tabla 6. Variación de la frecuencia Cardiaca Postpremedicación Midazolam oral (VO) vs intramuscular (IM) en los niños de 1-7 años en los niños de 1-7 años, grupo Midazolam oral VS Midazolam intramuscular.

\begin{tabular}{cccc}
\hline $\begin{array}{c}\text { Frecuencia } \\
\text { cardiaca }\end{array}$ & $\begin{array}{c}\text { Midazolam VO } \\
\text { MED } \pm \text { DE }\end{array}$ & $\begin{array}{c}\text { Midazolam IM } \\
\text { MED } \pm \text { DE }\end{array}$ & $\mathbf{p}$ \\
FC 0 & $94,33 \pm 10,67$ & $93,00 \pm 10,05$ & 0,48 \\
FC 5 & $94,41 \pm 10,66$ & $96,66 \pm 10,15$ & 0,23 \\
FC 10 & $95,16 \pm 10,57$ & $96,00 \pm 10,24$ & 0,66 \\
FC 15 & $95,25 \pm 10,06$ & $95,08 \pm 9,32$ & 0,92 \\
FC 30 & $94,83 \pm 10,16$ & $94,08 \pm 9,41$ & 0,67 \\
FC 45 & $93,66 \pm 10,33$ & $93,16 \pm 8,73$ & 0,77 \\
\hline
\end{tabular}

de administración. El análisis de las variaciones de la frecuencia cardiaca, no reveló diferencias significativas a los 5, 10, 15 y 30 minutos respecto del dato basal. Estos resultados concuerdan con los de otros autores quienes encontraron una mínima variación de la frecuencia cardiaca manteniéndose buena estabilidad hemodinámica ${ }^{(6,7)}$.

Por otro lado nuestros resultados, revelaron, que la presión arterial tampoco presentó diferencias significativas, lo que concuerda con estudios anteriores ${ }^{(3,5)}$.

En cuanto a la sedación consciente no encontramos diferencia significativa entre ambas vías de administración, lo que nos indica que ambas son buenas para tratar la ansiedad preoperatoria en niños.

Al evaluar la respuesta psicológica a la premedicación, los niños del grupo que recibieron midazolam intramuscular presentaron miedo al daño corporal (100\%) por el uso de agujas y jeringas. $(p<0,05)$. Estos resultados concuerdan con estudios previos de Suarez C, Morales Valdivia B ${ }^{(5)}$ (2010), y de Amundaraín, Meneses y Rodríguez ${ }^{(4)}$ (2004), 
que encontraron que los pacientes se desprendían fácilmente de sus padres.

Del mismo modo, los estudios de Riva Juan, Leibusiewicz, $\mathrm{G}^{(8)}$ et al (1998), demuestran que el uso del midazolam oral en pediatría proporciona buena sedación e inducción anestésica y que el sabor amargo lo mejoraron agregándole jugo de uva más edulcorante pues modificaba poco el $\mathrm{pH}$ de la solución y ocultaba dicho sabor amargo.

En nuestro estudio el midazolam vía oral si bien no presentó diferencias estadísticamente significativas en lo referente a la sedación consciente alcanzada, comparado con la vía intramuscular, es una buena alternativa para controlar la ansiedad de los niños; no hubieron diferencias significativas en cuanto a variaciones de las funciones hemodinámicas, lo que nos mostró que tiene buen perfil de seguridad para nuestros niños. Proporcionó la ventaja de no sumar temor y ansiedad a nuestro pequeño paciente antes de ingresar a sala de operaciones.

Nuestros resultados corroboran los estudios de autores nacionales $(2,9,10,11,12,13)$ e internacionales $(4,6,7,8,14,15$, 16,17) que sugieren el uso del midazolam oral en la premedicación anestésica de niños, comprobando su seguridad y eficacia en sedación consciente; buena aceptación oral de la pre-medicación, facilidad de administración, ausencia de miedo al daño corporal que afecte el estado psicológico del niño; desprendimiento fácil de los familiares y menor riesgo.

Fuentes de financiamiento: autofinanciado.

Conflicto de intereses: los autores declaran no tener conflictos de interés.

\section{Correspondencia}

María Luz Mateo Corrales

Urb. Los Ficus B-12. Ica, Perú.

Correo electrónico: blajere@hotmail.com

\section{REFERENCIAS BIBLIOGRÁFICAS}

1. Aguero-Peña RE, Amaranto MN, Sivira G, Mosquera MG, Valera E, García S, Pascuzzo-Lima C. Comparison of Midazolam, Bromazepam and Diazepam as oral premedication for patients undergoing elective surgery. Boletín Médico de Postgrado, 2006; 22(1): $1-2$.

2. Aldrete J.A, Guevara LU, Capmourteres E Texto de Anestesiología Teórico-Práctico. 2da. Edición. México D.F. Salvat. 2004. 1086- 1088

3. Castro ES, Díaz-Pizán ME, Valdiviezo M. Comparación de la efectividad del midazolam en niños: vía oral y vía intranasal. Rev. Estomatológica Herediana 2005; 15 (2): 133 - 138.

4. Amundaraín A, Meneses R, Rodríguez L, Mago A. Efectividad del jarabe de Midazolam para premedicación oral en pediatría. Archivos Venezolanos de Puericultura y Pediatría.2004; $67(2)$.
5. Suarez C, Morales B. Ventajas de la premedicación con midazolam en gomitas en niños. Actas Peruanas de Anestesiología. Revista de la Sociedad de Anestesia, Analgesia y Reanimación. 2010; 18(1).

6. Martinez H, Vera R, Flores J. Medicación Preanestésica con Midazolam Vía Oral en Niños. Revista Venezolana de Anestesiología. 1996; 1(1): 16-19.

7. Pérez AR. Medicación pre-anestésica con midazolam vía oral vs intranasal en preescolares. Hospital pediátrico Agustín Zubillaga. Tesis para optar el grado de Especialista en Anestesiología. Universidad Centro Occidental "Lisandro Alvarado" Barquisimeto 2001.

8. Riva J, Leibusiewicz G, Papa M, Lauber C. Oral Premedication With Midazolam In Pediatric Anaeshtesia. Effects On Sedation And Gastric Contents. Paediatr Anaesth. 1997; 7(3):191-6.

9. Cote $\mathbf{C H}$, Ryan J, Todres J, Goudaouzan N. Anestesia en Pediatría.2da. Edición: Ciudad de México. Interamericana. 1995. 141-156.

10. Castilla Moreno M. Manual de Anestesiología Pediátrica Para Médicos Residentes. 1ra Edición España. El Manual Moderno. 2001.

11. Kain Z, Sevarino F, Pincus S, et al. Attenuation of the Preoperative stress response with midazolam. Anesthesiology. . 2000: 93 (1):141-7

12. Miller, R. Anestesia. 4ta. Edición. Madrid. Harcourt Brace. 1998. 989-1016.

13. Paladino A, Masri L. Uso racional de las benzodiacepinas y su antagonista en anestesiología en Farmacología para Anestesiólogos e Intensivistas 2006. Argentina. Fundación Anestesiológica de Rosario.

14. Chávez EJ, Cortéz E, Hinojosa A, Araiza MA. Estudio comparativo de la premedicación con midazolam y ketamina por vía oral en pacientes pediátricos sometidos a tratamiento maxilofacial. Revista Odontológica Mexicana. 2005; 9(3): 131136.

15. Campin LR. Premedicación anestésica con Midazolam oral en pacientes preescolares del hospital Pediátrico Dr. Agustín Zubillaga. Tesis para optar el grado de Especialista en Anestesiología. Universidad Centro Occidental "Lisandro Alvarado" Barquisimeto 2001.

16. Gomero FG; Arrese FM, Vela L, Ruz G. Estudio comparativo de Midazolam en medicación preanestésica pediátrica vía oral vs. vía nasal en el Hospital Nacional Guillermo Almenara Irigoyen 1996 - 1997. Rev. Per. Anest. 1997; 10(2): 17-22. 
17. Pineda V, Dector T, Lopez M. Premedicación oral en niños, una comparación de dos dosificaciones de midazolam en cirugía ambulatoria. Revista Mexicana de Anestesiología. 1994; 17(4): 177- 82.

Recibido: 14/04/2012

Aceptado para publicación:22/07/2012 